\title{
An environmentally benign one pot synthesis of substituted quinolines catalysed by fluoroboric acid based ionic liquid
}

\author{
A RAJENDRAN $^{\mathrm{a}, *}, \mathrm{C} \mathrm{KARTHIKEYAN}^{\mathrm{b}}, \mathrm{K}^{\mathrm{RAJATHI}}{ }^{\mathrm{b}}$ and D RAGUPATHY \\ ${ }^{a}$ Department of Chemistry, Sir Theagaraya College, Chennai 600 021, India \\ ${ }^{\mathrm{b}}$ Research and Development Centre, Bharathiar University, Coimbatore 641 046, India \\ ${ }^{c}$ Department of Applied Chemistry, Kyungpook National University, 1370, Sangyuk 3 Dong, BUk GU, \\ Daegu 701-702, South Korea \\ e-mail: annamalai_rajendran2000@yahoo.com
}

MS received 23 September 2011; revised 13 January 2012; accepted 7 February 2012

\begin{abstract}
Organic synthesis generally required large amount of solvent, avoiding the use of organic solvents in synthesis is a paradigm shift directed at developing more benign chemistry, and with ionic liquids surprisingly can lead to access to new compounds. An elegant one-pot synthesis of quinoline derivatives has been achieved by reaction of substituted anilines with $\beta$-ketoester at $60^{\circ} \mathrm{C}$ in ethanol using an ionic liquid $\left[\mathrm{Et}_{3} \mathrm{NH}\right]^{+}\left[\mathrm{BF}_{4}\right]^{-}$as catalyst. All the reactions gave products with high degree of purity and excellent yield (78-93\%) within the shorter span of time (20-65 $\mathrm{min}$ ) than those reactions with conventional methods. The screening of solvents as well as the reuse of ionic liquid has been evaluated. The structure of the products has been elucidated by spectral and analytical data. The present scope and potential economic impact of the reaction are demonstrated by the synthesis of substituted quinolines. Remaining challenges and future perspectives of the new transformation are discussed.
\end{abstract}

Keywords. One-pot synthesis; benign synthesis; ionic liquids; fluoroboric acid; quinolines.

\section{Introduction}

In recent years, environment-friendly reaction processes have extensively been studied from the stand point of green chemistry. For example, oxidation reactions with the air, or reaction in water, supercritical fluids, and fluorous solvents are cited. ${ }^{1}$ Most recently, ionic liquids have gained much attention as green solvents for organic synthesis. The development of environmentally improved new synthetic routes, which are as much direct as possible and make use of safe and non-toxic starting materials, is a major target of the modern chemistry of organic synthesis. ${ }^{2}$ In recent years, the interest in room temperature ionic liquids is increasing as green reaction media for synthetic organic chemistry. Researchers have recently found that such ionic liquids are very useful as solvents as well as catalysts for several organic and inorganic syntheses. ${ }^{3}$ Ionic liquids are good candidates for replacement of toxic and volatile organic compounds because of their lower vapour pressures and lack of flammability. ${ }^{4-6}$ The synthesis of these ionic liquids (ILs), their characterization and possible applications have been developing progressively as the properties of this class of organic salts

*For correspondence with melting points below the boiling point of water have gained intensive attention in nearly all fields of chemistry. ${ }^{7}$ Furthermore, through the incorporation of functional groups, the synthesis of task specific ionic liquids (TSILs) has been a focus of research, leading to tailor-made substances for desired applications. Traditional chemical synthesis focused on optimizing yields, with little regard to a chemical's impact on the environment and its long term viability. There is now a realization that more benign chemical synthesis is required, as an integral part of developing sustainable technologies. Optimizing the yield is important but other issues need to be addressed, including minimizing the number of steps, simplicity, waste, atom efficiency, energy usage, safety and eco-friendliness. ${ }^{8}$

Lewis acidic ionic liquids have been receiving extensive interest as green substitute for $\mathrm{H}_{2} \mathrm{SO}_{4}, \mathrm{HF}$ and $\mathrm{AlCl}_{3}$ catalysts in chemical processes. Lewis acids are increasingly used in organic synthesis and have received considerable attention as non-toxic, recyclable and readily available catalysts for various organic transformations, affording the corresponding products in excellent yields with high selectivity. For many years, the synthesis of quinoline and its derivatives has been of considerable interest in organic and medicinal chemistry since a large number of natural products and drugs 
contain this heterocyclic moiety. In addition, quinolines are valuable synthons, used for the preparation of nano and meso structures with enhanced electronic and photonic properties. ${ }^{9}$ As part of the continuing effort towards the expeditious synthesis of biodynamic heterocycles, the possibility of developing a novel and efficient method to construct the quinoline scaffold became the focus of interest. ${ }^{10}$ This is inspite of some methods such as the Skraup, Doebner-von Miller, Combes reaction and Friedlander being valuable for the synthesis of quinolines. ${ }^{11-13}$ Bronsted acids and several Lewis acids have been reported to be effective for the synthesis of quinolines. However, many of these procedures also suffered from harsh reaction conditions (such as high temperature $>100^{\circ} \mathrm{C}$, use of litres of corrosive solvents even for milligram scale reactions and catalysts, time consumption, inability to recover the solvent and catalysts after use) low yields, difficult work-up and in some cases high catalyst loading had to be employed in order to obtain a respectable yield. ${ }^{14}$ The aforementioned drawbacks of conventional methods can be overcome when we use the ionic liquid $\left[\mathrm{Et}_{3} \mathrm{NH}\right]^{+}\left[\mathrm{BF}_{4}\right]^{-}$. Thus, simple, eco-benign and efficient procedures for the synthesis of these important heterocycles are still in demand. ${ }^{15}$ Inspired by reports on catalytic applications of fluoroboric acid for organic transformation and the endeavour of this research group toward the development of new synthetic methods, we report here a novel and efficient procedure for the synthesis of quinolines. ${ }^{16-18}$

\section{Experimental}

The melting points were determined in open capillary tubes in infra tech melting point apparatus and are uncorrected. ${ }^{19}$ The homogeneity of the products was checked on TLC plates coated with silica gel$\mathrm{G}$ and visualized by exposure to iodine vapours. ${ }^{20-23}$ The IR spectra were recorded on Perkin-Elmer Infrared model S99-B and on Shimadzu IR-435 spectrophotometer $\left(v_{\max }\right.$ in $\left.\mathrm{cm}^{-1}\right) .{ }^{1} \mathrm{H}$ NMR spectra were recorded on a Varian unity $250 \mathrm{MHz}$ NMR spectrometer using
TMS as an internal standard. ${ }^{24}$ Mass spectra were recorded on a JEOL SX 102/DA-6000 mass spectrometer. All reactants were purchased from SigmaAldrich and Lancaster and used as received. Solvents used in the reaction are double distilled by vacuum distillation. ${ }^{25-27}$

\subsection{General procedure for the synthesis of substituted quinolines, $\mathbf{3 a - h}$}

In a $50 \mathrm{ml}$ round bottom flask, substituted anilines 1ah $(1 \mathrm{mmol})$, ethyl acetoacetate $2(1 \mathrm{mmol})$ and $5 \mathrm{~mol} \%$ $\left[\mathrm{Et}_{3} \mathrm{NH}\right]^{+}\left[\mathrm{BF}_{4}\right]^{-}$in ethanol $(15 \mathrm{ml})$ were mixed and the resulting solution was stirred at $60^{\circ} \mathrm{C}$. After completion of the reaction as monitored by TLC, the reaction mixture was filtered and the crude product was subjected to purification by flash chromatography using a mixture of $20 \%$ ethyl acetate and $80 \% n$-hexane as eluent to yield the quinolines $\mathbf{3 a - h}$. The purified product was recrystallized using appropriate solvent. The structure of all the products was unambiguously established on the basis of their spectral analysis.

\section{Results and discussion}

An initial study was performed by the treatment of aniline $\mathbf{1 a}$ with ethyl acetoacetate $\mathbf{2}$ in methanol in the presence of catalytic amounts of $\left[\mathrm{Et}_{3} \mathrm{NH}\right]^{+}\left[\mathrm{BF}_{4}\right]^{-}(10 \mathrm{~mol}$ $\%)$ at RT (scheme 1).

To our delight, the formation of 2-hydroxy-4-methylquinoline 3a was observed. Complete conversion and $93 \%$ isolated yields were obtained after $30 \mathrm{~min}$. Further studies established that $5 \mathrm{~mol} \%$ of catalyst was equally efficient to perform the reaction (table 1).

Moreover, it is noteworthy that this reaction could be run under normal condition without loss in efficiency. Among the solvent screened, ethanol and methanol were demonstrated as the best solvent. Further procedures were developed with ethanol as it is more environmentally benign as compared to methanol. This is because of high solubility of $\left[\mathrm{Et}_{3} \mathrm{NH}\right]^{+}\left[\mathrm{BF}_{4}\right]^{-}$in polar solvents. Under solvent-free conditions, the reaction

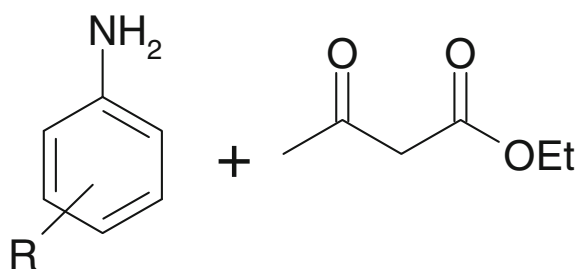

1a-h
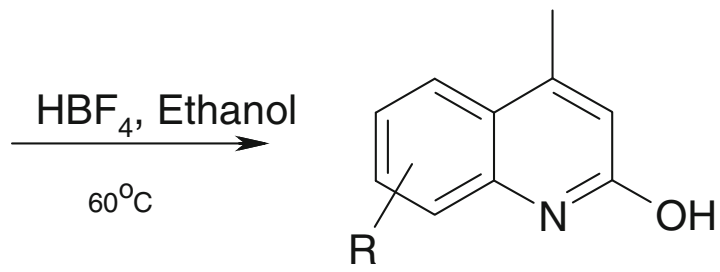

3a-h

Scheme 1. Synthesis of substituted quinolines. 
Table 1. Evaluation of catalytic activity of $\left[\mathrm{Et}_{3} \mathrm{NH}\right]^{+}\left[\mathrm{BF}_{4}\right]^{-}$ on the synthesis of various substituted-4-methyl quinoline2-ol.

\begin{tabular}{lccc}
\hline Entry & $\begin{array}{c}{\left[\mathrm{Et}_{3} \mathrm{NH}\right]^{+}\left[\mathrm{BF}_{4}\right]^{-}} \\
(\mathrm{X} \mathrm{mol} \%)\end{array}$ & $\begin{array}{c}\text { Time } \\
(\min )\end{array}$ & ${ }^{b}$ Yield(\%) \\
\hline 1 & 0 & 60 & 0 \\
2 & 5 & 45 & 88 \\
3 & 10 & 30 & 93 \\
4 & 15 & 25 & 93 \\
5 & 20 & 20 & 95 \\
\hline
\end{tabular}

Reactions conditions: aniline (1a, $1 \mathrm{mmol})$; ethylacetoacetate $(2,1 \mathrm{mmol}) ;\left[\mathrm{Et}_{3} \mathrm{NH}\right]^{+}\left[\mathrm{BF}_{4}\right]^{-}(\mathrm{X} \mathrm{mol} \%)$; solvent ethanol; RT; 1 atm. ${ }^{\mathrm{b}}$ Isolated yields

also proceeded smoothly to afford the corresponding product, although the yield was slightly lower (table 2).

In order to examine the effect of temperature, concentration of $\left[\mathrm{Et}_{3} \mathrm{NH}\right]^{+}\left[\mathrm{BF}_{4}\right]^{-}$was kept constant at $5 \mathrm{~mol} \%$ and the reaction was monitored at different temperatures as compiled in table 3. At elevated temperatures using $5 \mathrm{~mol} \%$ of $\left[\mathrm{Et}_{3} \mathrm{NH}\right]^{+}\left[\mathrm{BF}_{4}\right]^{-}$gave better results in terms of yield and reaction times. To demonstrate the generality of this method, the scope of this reaction under optimized conditions was investigated and it was found that structurally diverse aromatic amines with different substitution (both electron withdrawing and electron donating group) reacted with equal ease to produce a range quinolines derivatives in excellent yields (table 4).

It is important to stress that the catalyst was recycled by solvent (water) extraction method and reused for five runs with only slight drop in activity (table 5).

An additional starting material was added into the reaction mixture and reaction was continued for an additional $10 \mathrm{~h}$ which resulted in the formation of $\mathbf{3 a}$ in excellent yields. The structure of the products $(\mathbf{3 a}-\mathbf{h})$ obtained was elucidated by ${ }^{1} \mathrm{H}$ NMR, FT-IR and Mass spectral studies. ${ }^{26}$

Table 2. Screening of solvents for the synthesis of various substituted 4-methylquinoline-2-ol.

\begin{tabular}{lccc}
\hline Entry & Solvent & Time (min) & ${ }^{\text {b } Y i e l d ~}(\%)$ \\
\hline 1 & Methanol & 45 & 88 \\
2 & Ethanol & 35 & 86 \\
3 & Dichloromethane & 70 & 64 \\
4 & Acetonitrile & 55 & 72 \\
5 & Solvent free condition & 40 & 70 \\
\hline
\end{tabular}

Reactions conditions: aniline (1a, $1 \mathrm{mmol})$; ethylacetoacetate $(2,1 \mathrm{mmol}) ;\left[\mathrm{Et}_{3} \mathrm{NH}\right]^{+}\left[\mathrm{BF}_{4}\right]^{-}(\mathrm{X} \mathrm{mol} \%)$; solvent ethanol; RT; 1 atm. ${ }^{\text {b Isolated yields }}$
Table 3. Effect of temperature on the synthesis of various substituted-4-methylquinoline-2-ol.

\begin{tabular}{lccc}
\hline Entry & Temperature $\left({ }^{\circ} \mathrm{C}\right)$ & Time $(\min )$ & ${ }^{\mathrm{b}}$ Yield $(\%)$ \\
\hline 1 & RT & 35 & 86 \\
2 & 45 & 30 & 90 \\
3 & 60 & 25 & 93 \\
4 & $\geq 80$ & 20 & 94 \\
\hline
\end{tabular}

${ }^{\mathrm{b}}$ Isolated yield based upon the starting amine

\subsection{Spectral data}

Compound 3a: M.F.C ${ }_{10} \mathrm{H}_{9} \mathrm{NO} ;{ }^{1} \mathrm{H}$ NMR - $2.14 \delta$ $\left(\mathrm{s}, 3 \mathrm{H}, \mathrm{CH}_{3}\right), 6.15 \delta(\mathrm{s}, 1 \mathrm{H}, \mathrm{CH}), 7.33-7.4 \delta(\mathrm{m}$, $4 \mathrm{H}), 8.43 \delta(\mathrm{s}, 1 \mathrm{H}, \mathrm{NH})$; IR- $3400 \mathrm{~cm}^{-1}(\mathrm{O}-\mathrm{H}, \mathrm{str})$, $1650 \mathrm{~cm}^{-1}(\mathrm{C}=\mathrm{N}, \mathrm{str}), 3030 \mathrm{~cm}^{-1}(\mathrm{C}-\mathrm{H}, \mathrm{str}$, aromatic $)$, $1100 \mathrm{~cm}^{-1}(\mathrm{C}-\mathrm{C}, \mathrm{str}) ; 1650 \mathrm{~cm}^{-1}(\mathrm{C}=\mathrm{C}$, str); Mass- MS: $\mathrm{m} / \mathrm{z}\left[\mathrm{M}^{+}\right] 159$

Compound 3b: M.F. $\mathrm{C}_{10} \mathrm{H}_{8} \mathrm{FNO} ;{ }^{1} \mathrm{H}$ NMR $-2.28 \delta$ $\left(\mathrm{s}, 3 \mathrm{H}, \mathrm{CH}_{3}\right), 6.17 \delta(\mathrm{s}, 1 \mathrm{H}, \mathrm{CH}), 7.22 \delta(\mathrm{s}, 1 \mathrm{H}, \mathrm{CH})$, $7.53 \delta(\mathrm{d}, 1 \mathrm{H}, \mathrm{J}=7.7 \mathrm{~Hz}, \mathrm{CH}), 7.66 \delta(\mathrm{d}, 1 \mathrm{H}, \mathrm{J}=$ $7.6 \mathrm{~Hz}, \mathrm{CH}), 9.12 \delta(\mathrm{s}, 1 \mathrm{H}, \mathrm{NH})$; IR- $3400 \mathrm{~cm}^{-1}(\mathrm{O}-\mathrm{H}$, str), $1650 \mathrm{~cm}^{-1}(\mathrm{C}=\mathrm{N}, \mathrm{str}), 3030 \mathrm{~cm}^{-1}(\mathrm{C}-\mathrm{H}, \mathrm{str}$, aromatic), $1100 \mathrm{~cm}^{-1}(\mathrm{C}-\mathrm{C}, \mathrm{str}) ; 1650 \mathrm{~cm}^{-1}(\mathrm{C}=\mathrm{C}$, str), $1300 \mathrm{~cm}^{-1}(\mathrm{C}-\mathrm{F}, \mathrm{str})$; Mass- MS: m/z $\left[\mathrm{M}^{+}\right] 175$

Compound 3c: $\mathrm{C}_{11} \mathrm{H}_{11} \mathrm{NO} ;{ }^{1} \mathrm{H}$ NMR $-2.28 \delta(\mathrm{s}$, $\left.3 \mathrm{H}, \mathrm{CH}_{3}\right), 2.43 \delta\left(\mathrm{S}, 3 \mathrm{H}, \mathrm{CH}_{3}\right), 7.25 \delta(\mathrm{s}, 1 \mathrm{H}, \mathrm{CH})$, $7.27 \delta(\mathrm{s}, 1 \mathrm{H}, \mathrm{CH}), 7.51 \delta(\mathrm{d}, 1 \mathrm{H}, \mathrm{J}=7.7 \mathrm{~Hz}$, $\mathrm{CH}), 7.54 \delta(\mathrm{d}, 1 \mathrm{H}, \mathrm{J}=7.6 \mathrm{~Hz}, \mathrm{CH}), 9.10 \delta(\mathrm{s}, 1 \mathrm{H}$, $\mathrm{NH})$; IR- $3400 \mathrm{~cm}^{-1}(\mathrm{O}-\mathrm{H}, \mathrm{str}), 1650 \mathrm{~cm}^{-1}(\mathrm{C}=\mathrm{N}, \mathrm{str})$, $3030 \mathrm{~cm}^{-1}$ (C-H, str, aromatic), $1100 \mathrm{~cm}^{-1}(\mathrm{C}-\mathrm{C}, \mathrm{str})$; $1650 \mathrm{~cm}^{-1}(\mathrm{C}=\mathrm{C}, \mathrm{str}), 3100 \mathrm{~cm}^{-1}(\mathrm{C}-\mathrm{H}$, str, aliphatic); Mass- MS: m/z $\left[\mathrm{M}^{+}\right] 173$

Compound 3d: $\mathrm{C}_{11} \mathrm{H}_{11} \mathrm{NO}_{2} ;{ }^{1} \mathrm{H}$ NMR - $2.38 \delta(\mathrm{S}$, $\left.3 \mathrm{H}, \mathrm{CH}_{3}\right), 3.82 \delta\left(\mathrm{S}, 3 \mathrm{H}, \mathrm{CH}_{3}\right), 6.43 \delta(\mathrm{s}, 1 \mathrm{H}, \mathrm{CH}), 7.52$ $\delta(\mathrm{s}, 1 \mathrm{H}, \mathrm{CH}), 7.51 \delta(\mathrm{d}, 1 \mathrm{H}, \mathrm{J}=7.7 \mathrm{~Hz}, \mathrm{CH}), 7.63$ $\delta(\mathrm{d}, 1 \mathrm{H}, \mathrm{J}=7.6 \mathrm{~Hz}, \mathrm{CH}), 7.81 \delta(\mathrm{d}, 1 \mathrm{H}, \mathrm{J}=7.6 \mathrm{~Hz}$, $\mathrm{CH}), 8.82 \delta(\mathrm{s}, 1 \mathrm{H}, \mathrm{NH})$; IR- $3400 \mathrm{~cm}^{-1}(\mathrm{O}-\mathrm{H}, \mathrm{str})$, $1650 \mathrm{~cm}^{-1}(\mathrm{C}=\mathrm{N}, \mathrm{str}), 3030 \mathrm{~cm}^{-1}(\mathrm{C}-\mathrm{H}, \mathrm{str}$, aromatic $)$, $1100 \mathrm{~cm}^{-1}$ (C-C, str), $1650 \mathrm{~cm}^{-1}(\mathrm{C}=\mathrm{C}, \mathrm{str}), 3100 \mathrm{~cm}^{-1}$, $1250 \mathrm{~cm}^{-1}$ (-O-C, str), $3100 \mathrm{~cm}^{-1}$ (C-H, str, aliphatic); MS: $\mathrm{m} / \mathrm{z}\left[\mathrm{M}^{+}\right] 189$

Compound 3e: $\mathrm{C}_{10} \mathrm{H}_{8} \mathrm{~N}_{2} \mathrm{O}_{3} ;{ }^{1} \mathrm{H}$ NMR - $2.25 \delta(\mathrm{s}$, $\left.3 \mathrm{H}, \mathrm{CH}_{3}\right), 3.82 \delta\left(\mathrm{S}, 3 \mathrm{H}, \mathrm{CH}_{3}\right), 6.14 \delta(\mathrm{s}, 1 \mathrm{H}, \mathrm{CH})$, $7.41 \delta(\mathrm{s}, 1 \mathrm{H}, \mathrm{CH}), 7.65 \delta(\mathrm{d}, 1 \mathrm{H}, \mathrm{J}=7.4 \mathrm{~Hz}, \mathrm{CH})$, $7.78 \delta(\mathrm{d}, 1 \mathrm{H}, \mathrm{J}=7.6 \mathrm{~Hz}, \mathrm{CH}), 8.42 \delta(\mathrm{s}, 1 \mathrm{H}$, $\mathrm{NH})$; IR- $3400 \mathrm{~cm}^{-1}\left(\mathrm{O}-\mathrm{H}\right.$, str), $1650 \mathrm{~cm}^{-1}(\mathrm{C}=\mathrm{N}, \mathrm{str})$, 
Table 4. $\left[\mathrm{Et}_{3} \mathrm{NH}\right]^{+}\left[\mathrm{BF}_{4}\right]^{-}$promoted synthesis of various substituted 4-methylquinoline-2-ol.

\begin{tabular}{lcccccc}
\hline Entry & Aniline & $\mathrm{R}$ & Time (min) & Product & ${ }^{\mathrm{b}}$ Yield $(\%)$ & m.p. $\left({ }^{\circ} \mathrm{C}\right)$ \\
\hline 1 & $\mathbf{1 a}$ & $\mathrm{H}$ & 20 & $\mathbf{3 a}$ & 93 & 218 \\
2 & $\mathbf{1 b}$ & $4-\mathrm{F}$ & 25 & $\mathbf{3 b}$ & 78 & 261 \\
3 & $\mathbf{1 c}$ & $4-\mathrm{CH}_{3}$ & 35 & $\mathbf{3 c}$ & 84 & 249 \\
4 & $\mathbf{1 d}$ & $4-\mathrm{OCH}_{3}$ & 30 & $\mathbf{3 d}$ & 91 & 257 \\
5 & $\mathbf{1 e}$ & $4-\mathrm{NO}_{2}$ & 65 & $\mathbf{3 e}$ & 76 & 300 \\
6 & $\mathbf{1 f}$ & $4-\mathrm{COOH}$ & 40 & $\mathbf{3 f}$ & 76 & 300 \\
7 & $\mathbf{1 g}$ & 2-pyridinyl & 30 & $\mathbf{3 g}$ & 80 & 181 \\
8 & $\mathbf{1 h}$ & 2-napthylamine & 45 & $\mathbf{3 h}$ & 87 & 271 \\
\hline
\end{tabular}

Reactions conditions: aniline (1a, $1 \mathrm{mmol})$; ethylacetoacetate $(\mathbf{2}, 1 \mathrm{mmol})$; $\left[\mathrm{Et}_{3} \mathrm{NH}\right]^{+}\left[\mathrm{BF}_{4}\right]^{-}(5 \mathrm{~mol} \%)$; solvent ethanol; temperature $60^{\circ} \mathrm{C} ; 1 \mathrm{~atm} .{ }^{b}$ Isolated yields

Table 5. Reuse of $\left[\mathrm{Et}_{3} \mathrm{NH}\right]^{+}\left[\mathrm{BF}_{4}\right]^{-}$in the synthesis of $3 \mathbf{a}$ using ethanol at $60^{\circ} \mathrm{C}$ for $20 \mathrm{~min}$.

\begin{tabular}{lc}
\hline Entry & Yield (\%) \\
\hline 1 & 91 \\
2 & 90 \\
3 & 90 \\
4 & 88 \\
5 & 87 \\
\hline
\end{tabular}

${ }^{\mathrm{b}}$ Isolated yield based upon the starting amine

$3030 \mathrm{~cm}^{-1}$ (C-H, str, aromatic), $1100 \mathrm{~cm}^{-1}$ (C-C, str); $1650 \mathrm{~cm}^{-1}(\mathrm{C}=\mathrm{C}, \mathrm{str}), 3100 \mathrm{~cm}^{-1}, 1560 \mathrm{~cm}^{-1}(\mathrm{C}-\mathrm{N}, \mathrm{str}$,

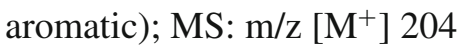

Compound 3f: $\mathrm{C}_{11} \mathrm{H}_{9} \mathrm{NO}_{3} ;{ }^{1} \mathrm{H}$ NMR - $2.41 \delta(\mathrm{s}$, $\left.3 \mathrm{H}, \mathrm{CH}_{3}\right), 6.44 \delta(\mathrm{s}, 1 \mathrm{H}, \mathrm{CH}), 7.88-8.96 \delta(\mathrm{m}, 3 \mathrm{H}$, $\mathrm{CH}), 7.83 \delta(\mathrm{d}, 1 \mathrm{H}, \mathrm{J}=7.3 \mathrm{~Hz}, \mathrm{CH}), 9.46 \delta(\mathrm{s}, 1 \mathrm{H}$, $\mathrm{NH}), 11.36 \delta(\mathrm{s}, 1 \mathrm{H}, \mathrm{COOH})$; IR- $3400 \mathrm{~cm}^{-1}(\mathrm{O}-\mathrm{H}$, str), $1650 \mathrm{~cm}^{-1}(\mathrm{C}=\mathrm{N}, \mathrm{str}), 3030 \mathrm{~cm}^{-1}(\mathrm{C}-\mathrm{H}$, str, aromatic), $1100 \mathrm{~cm}^{-1}(\mathrm{C}-\mathrm{C}, \mathrm{str}) ; 1650 \mathrm{~cm}^{-1}(\mathrm{C}=\mathrm{C}, \mathrm{str})$, $1750 \mathrm{~cm}^{-1}(\mathrm{C}=\mathrm{O}$, str of $-\mathrm{COOH}$ group $)$; MS: $\mathrm{m} / \mathrm{z}\left[\mathrm{M}^{+}\right]$ 203

Compound 3g: $\mathrm{C}_{12} \mathrm{H}_{12} \mathrm{~N}_{2} \mathrm{O} ;{ }^{1} \mathrm{H}$ NMR - $2.41 \delta$ (s, $\left.3 \mathrm{H}, \mathrm{CH}_{3}\right), 6.44 \delta(\mathrm{s}, 1 \mathrm{H}, \mathrm{CH}), 7.88-8.96 \delta(\mathrm{m}, 3 \mathrm{H}$, $\mathrm{CH}), 7.83 \delta(\mathrm{d}, 1 \mathrm{H}, \mathrm{J}=7.3 \mathrm{~Hz}, \mathrm{CH}), 9.46 \delta(\mathrm{s}, 1 \mathrm{H}$, $\mathrm{NH}), 11.36 \delta(\mathrm{s}, 1 \mathrm{H}, \mathrm{COOH})$; IR- $3400 \mathrm{~cm}^{-1}(\mathrm{O}-\mathrm{H}$, str), $1650 \mathrm{~cm}^{-1}(\mathrm{C}=\mathrm{N}$, str $), 3030 \mathrm{~cm}^{-1}(\mathrm{C}-\mathrm{H}$, str, aromatic), $1100 \mathrm{~cm}^{-1}(\mathrm{C}-\mathrm{C}, \mathrm{str}) ; 1650 \mathrm{~cm}^{-1}(\mathrm{C}=\mathrm{C}$, str), $1300 \mathrm{~cm}^{-1}(\mathrm{C}-\mathrm{N}$, str of pyridinyl); MS: m/z [M+ 236

Compound 3h: $\mathrm{C}_{20} \mathrm{H}_{15} \mathrm{~N}_{2} \mathrm{O} ;{ }^{1} \mathrm{H}$ NMR $-2.33 \delta$ (s, $\left.3 \mathrm{H}, \mathrm{CH}_{3}\right), 6.03 \delta(\mathrm{s}, 1 \mathrm{H}, \mathrm{CH}), 7.22-7.63 \delta(\mathrm{m}, 3 \mathrm{H}$, $\mathrm{CH}), 7.210-7.674 \delta(\mathrm{m}, 5 \mathrm{H}, \mathrm{CH}), 6.892-6.925 \delta(\mathrm{m}$,
$\mathrm{H}, \mathrm{CH}), 3.76 \delta\left(\mathrm{s}, 2 \mathrm{H}, \mathrm{NH}_{2}\right)$; IR- $3400 \mathrm{~cm}^{-1}(\mathrm{O}-\mathrm{H}$, str), $1650 \mathrm{~cm}^{-1}(\mathrm{C}=\mathrm{N}, \mathrm{str}), 3030 \mathrm{~cm}^{-1}(\mathrm{C}-\mathrm{H}, \mathrm{str}$, aromatic), $1100 \mathrm{~cm}^{-1}(\mathrm{C}-\mathrm{C}, \mathrm{str}) ; 1650 \mathrm{~cm}^{-1}(\mathrm{C}=\mathrm{C}$, str), $3400 \mathrm{~cm}^{-1}(\mathrm{~N}-\mathrm{H}, \mathrm{str}) ; \mathrm{MS}: \mathrm{m} / \mathrm{z}\left[\mathrm{M}^{+}\right] 300$

\section{Conclusion}

In conclusion, we have described a simple, efficient and a facile tandem protocol for an elegant one-pot synthesis of quinoline derivatives by reaction of substituted anilines with $\beta$-ketoester at $60^{\circ} \mathrm{C}$ in ethanol using fluoroboric acid / $\left[\mathrm{Et}_{3} \mathrm{NH}\right]^{+}\left[\mathrm{HSO}_{4}\right]^{-}$as catalyst. The major advantages of the present method are much faster reaction, easy work up procedure and good to excellent yields and avoiding the usage of hazardous organic solvent and toxic catalyst. In addition to this, the fluoroboric acid / $\left[\mathrm{Et}_{3} \mathrm{NH}\right]^{+}\left[\mathrm{HSO}_{4}\right]^{-}$catalyst was successfully reused for five runs without potential loss of activity. This proves that this method is much more convenient than those with conventional catalysts.

\section{Acknowledgements}

AR acknowledges the funding support given by the University Grants Commission, New Delhi for his major research project [F.No 35-147/2009(SR)]. He thanks the principal and the management of $\mathrm{Sir}$ Theagaraya College, Chennai for the constant encouragement. His thanks are also due to Mr. P Sakthivel, Sastra University, Tanjore for the spectral recording.

\section{References}

1. Beckman E J 2004 J. Supercritical Fluids 28121

2. Ghosh R 2001 Indian J. Chem. 40B 550

3. Ranu B C 2000 Eur. J. Org. Chem. 282347 
4. Katrizky A R and Rees C N 1984 Comprehensive Heterocyclic Chemistry; A J Boulton (ed.); Pergamon: Oxford, Vol. 2. pp.385

5. Agrawal A K and Jenekhe S A 1991 Macromolecules 24 6806

6. Zhang X, Shetty A S and Jenekhe S A 2001 Macromolecules 347315

7. Jenekhe S A, Lu L and Alam M M 2001 Macromolecules 34P 7315

8. Du W and Curran D P 2003 Org. Let. 51765

9. Hoemann M Z, Kumaravel G, Xie R L, Rossi R F, Meyer S, Sidhu A, Cuny G D and Hauske J R 2000 Bioorg. Med. Chem. Lett. 10L 2675

10. Kidwai M, Bansal V, Saxena A, Aerry S and Mozumdar S 2006 Tetrahedron Lett. 478049

11. Kidwai M, Mothsra P, Mohan R and Biswas S 2005 Bioorg. Med. Chem. Lett. 15915

12. Cho C S, Oh B H, Kim J S, Kim T J and Shim S C 2000 Chem. Commun. 161885

13. Jiang B and Si Y G 2002 J. Org. Chem. 679449

14. Skraup Z H 1980 Chem. Ber. 132086

15. Friedlander P 1982 Chem. Ber. 152572
16. Mansake R H F and Kulka M 1953 Org. React. 7P 59

17. Wu J, Xia H G and Gao K 2006 Org. Biomol. Chem. 4 126

18. Strekowski L, Czrany A and Lee H 2000 J. Flourine Chem. 104281

19. Hu Y Z, Zhang G and Thummel R P 2003 Org. Lett. 5 2251

20. Arcade A, Chiarini M, Di Giuseppe S and Marinelli F 2003 Syn. lett. 203

21. Walser A, Flynn T and Fryer R I 1975 J. Heterocycl. Chem. 12737

22. Arumugam P, Karthikeyan G, Atchudan R, Muralidharan D and Perumal P T 2005 Chem. Lett. 34314

23. Wu J, Zhang L and Diao T N 2005 Syn. lett. 52653

24. Kidwai M, Bansal V, Kumar A and Mozumdar S 2007 Green Chem. 9742

25. Kidwai M, Mishra N K, Bansal V, Kumar A and Mozumdar S 2007 Tetrahedron Lett. 488883

26. Kidwai M, Bansal V, Mishra N K, and Bhatnagar D 2009 Indian J. Chem. 48B 746

27. Cheng 1982 Organic Reactions 28348 